\title{
Diversidad, modelos de gestión y formación inicial docente: desafíos formativos desde una perspectiva de justicia social*
}

FELIPE JIMÉNEZ VARGAS

Pontificia Universidad Católica de Valparaíso, Viña del Mar, Chile

CARMEN MONTECINOS SANHUEZA

Pontificia Universidad Católica de Valparaíso, Viña del Mar, Chile

\section{RESUMEN}

El estudio examina enfoques con que ocho profesores novatos gestionan la diversidad escolar y la relación entre estos y su formación inicial. La producción de datos incluyó 12 entrevistas y 20 observaciones participantes en las aulas de estos docentes. Los resultados evidencian dos enfoques de gestión de la diversidad: uno centrado en las necesidades educativas especiales del alumnado, y otro orientado al fortalecimiento de relaciones humanas en la escuela. A su vez, se evidencia una insuficiente formación pedagógica que desarrolle las competencias para trabajar en escenarios educativos diversos. La discusión evidencia que estos enfoques corresponden a modalidades reduccionistas de gestión, debido a la falta de conexión con una perspectiva de justicia social. Finalmente, se ofrecen recomendaciones para orientar la formación inicial hacia una perspectiva inclusiva y socialmente justa de los procesos educativos.

\section{PALABRAS CLAVE}

desigualdades educativas; atención a la diversidad; justicia social; formación inicial docente; profesores noveles.

* Investigación financiada por Comisión Nacional de Investigación Científica y Tecnológica (CONICYT)/ Fondo Nacional de Desarrollo Científico y Tecnológico (FONDECYT). Los resultados forman parte de los proyectos FONDECYT/POSTDOCTORADO n. 3140247 y FONDECYT/INICIACION n. 11150831. 


\title{
DIVERSITY, MANAGEMENT MODELS AND INITIAL TEACHER TRAINING: TRAINING CHALLENGES FROM THE PERSPECTIVE OF SOCIAL JUSTICE
}

\begin{abstract}
The study examines how eight novice teachers manage diversity in their classrooms and its relationship to their experiences during initial teacher preparation. Data production involved 12 interviews and 20 participant's observations in these teachers' classrooms. Findings show two approaches to managing diversity: one focused on students' special educational needs and another strengthening human relations in the school. Moreover, an inadequate preparation to develop the pedagogical skills to work in heterogeneous educational settings is evident. We discuss the shortcomings of these approaches to the extent that they lack a connection with a social justice perspective. Finally, recommendations are offered to guide initial teacher preparation toward inclusive and socially just educational processes.
\end{abstract}

\section{KEYWORDS}

educational inequalities; attention to diversity; social justice; initial teacher preparation; novice teachers.

\section{DIVERSIDADE, MODELOS DE GESTÃO E FORMAÇÃO DOCENTE INICIAL: DESAFIOS DE FORMAÇÃO COM BASE EM UMA PERSPECTIVA DE JUSTIÇA SOCIAL}

\section{RESUMO}

O estudo examina como oito professores novatos lidam com a diversidade em sala de aula, e a relação entre eles e sua formação inicial. A produção de dados incluiu 12 entrevistas e 20 observações partícipes nas aulas desses docentes. Os resultados evidenciam duas abordagens na gestão da diversidade: uma centrada nas necessidades educacionais especiais dos alunos, e outra orientada para o fortalecimento de relações humanas na escola. Por sua vez, torna-se evidente uma formação pedagógica insuficiente, que desenvolva as competências para trabalhar em cenários educacionais diversos. A discussão mostra que esses enfoques correspondem a modalidades reducionistas de gestão em virtude da falta de conexão com uma perspectiva de justiça social. Finalmente, são apresentadas recomendações para orientar a formação inicial do professor rumo a uma perspectiva inclusiva e socialmente justa dos processos educacionais.

\section{PALAVRAS-CHAVE}

desigualdades educacionais; atenção à diversidade; justiça social; formação inicial docente; professores novatos. 


\section{INTRODUCCIÓN}

Los cambios socioculturales que atraviesa nuestra sociedad así como cambios en materia de políticas educativas, han hecho de nuestras escuelas escenarios cada vez más diversos. El aumento sistemático de estudiantes inmigrantes en las escuelas producto de la intensificación de los flujos migratorios, así como el aumento progresivo de proyectos de integración escolar como reemplazo de las escuelas especiales, grafican cómo la composición del alumnado se hace cada vez más heterogénea. Sin embargo, como evidencian los recientes resultados de mediciones como Sistema de Medición de la Calidad de la Enseñanza (SIMCE) ${ }^{1}$ o Programa para la Evaluación Internacional de Alumnos (PISA), los desempeños de aprendizaje se presentan de manera significativamente desigual. Si observamos los resultados de PISA 2012, se puede apreciar que del conjunto de los 34 países participantes de la Organización para la Cooperación y el Desarrollo Económicos (OCDE), los resultados académicos de estudiantes inmigrantes son consistentemente inferiores - 42 puntos promedio - en comparación con los resultados del alumnado autóctono (OECD, 2013). En el caso de Chile, si bien los inmigrantes obtienen mejores resultados que el promedio de países de la OCDE, estos obtienen no obstante inferiores resultados que sus pares nativos (AGCE, 2014). Esto evidencia que al igual que estudiantes de determinados grupos desfavorecidos, ${ }^{2}$ los inmigrantes también se ven afectados por las desigualdades del sistema educativo. En este escenario, uno de los principales desafíos del sistema educativo chileno es resolver las profundas desigualdades de aprendizaje y participación que afecta a una parte significativa de sus estudiantes.

Si bien resolver las desigualdades educativas requiere abordar una perspectiva ecológica que incorpore las diferentes dimensiones y niveles que componen el sistema educativo, esta investigación, desde una perspectiva inclusiva y de justicia social en educación (Ainscow, 2004; Dover, 2009; Sleeter, 2013), sostiene que una manera privilegiada de aproximarse a la comprensión del fenómeno de las desigualdades educativas radica en la comprensión de los modelos y enfoques de gestión de la diversidad utilizados por el profesorado en las escuelas. Conocer con qué modelos gestionan la diversidad de sus aulas el profesorado y cuáles son sus implicancias educativas, se constituye en una valiosa fuente de información tanto para el mejoramiento de las prácticas pedagógicas cotidianas, como para el fortalecimiento de los programa de formación inicial en materia de diversidad en las escuelas.

Ahora bien, aun cuando en la actualidad existe abundante investigación en torno a la formación inicial docente en Chile (Avalos, 2002, 2006, 2009; Miranda, 2007; Montecinos, 2003), la mayoría de esta no ha estado orientada a comprender y analizar su rol en la preparación de futuros docentes para gestionar la diversidad desde una perspectiva inclusiva y de justicia social. En este contexto,

1 Prueba anual utilizada en Chile para medir y comparar los resultados de aprendizaje de los estudiantes en las escuelas.

2 Estudiantes pertenecientes a clases sociales en contextos de pobreza y/o pertenecientes a etnias indígenas principalmente. 
la presente investigación busca comprender los modelos de gestión de la diversidad utilizados por el profesorado de manera de conocer cómo estos están enfrentando el desafío de trabajar en contextos educativos cada vez más diversos y qué dificultades presentan para favorecer aprendizajes de calidad para todos sus estudiantes. El objetivo ulterior de la investigación es precisar los requerimientos y necesidades formativas del profesorado, de manera de entregar información útil a los programas de formación docente para la implementación de procesos de mejora.

\section{MARCO TEÓRICO}

\section{DIVERSIDAD Y FORMACIÓN INICIAL DOCENTE}

Pese a que la diversidad se ha ido constituyendo en un elemento estructural que ha hecho de nuestras escuelas espacios cada vez más heterogéneos (Jiménez, 2012), y pese a que diversas investigaciones han evidenciado la falta de herramientas del profesorado para trabajar bajo estas nuevas necesidades y demandas (Ibáñez, 2010; Jiménez y Fardella, 2015; Quilaqueo y Quintriqueo, 2008), los programas de formación docente en Chile no han respondido oportunamente a esta nueva problemática educativa.

A través de una revisión de las mallas curriculares de pedagogías de universidades pertenecientes al Consejo de Rectores de las Universidades Chilenas (CRUCH) en Chile, ${ }^{3}$ Venegas (2013) evidenció que del total de programas formativos, en solo cinco casos los currículos presentaron instancias formativas (asignatura, unidad y/o contenido) relacionadas con temáticas de diversidad: culturas originarias, educación rural, género, multiculturalismo y relación comunidad-sociedad. Esta evidencia permite concluir que si hay una perspectiva central a la hora de comprender las dificultades que para las escuelas está significando el desarrollar los procesos de escolarización con un alumnado cada vez más diverso, es la relacionada con la escasa formación inicial del profesorado en la materia.

Resultados recientes de investigaciones exploratorias en base a estudios de caso de programas formativos en Chile (Tenorio, 2011; Zapata, 2011), han evidenciado que tanto desde el punto de vista de los planes de estudio como de la percepción del profesorado egresado, las instancias de formación destinadas a abordar la diversidad son escasas. Zapata (2011), en una investigación realizada con 80 estudiantes pertenecientes a 10 carreras de pedagogía, reportó que prácticamente la totalidad de los participantes no reconocía fortalezas respecto a su formación, sino solo debilidades en materia de atención a la diversidad en el aula. Tenorio (2011), estudió la experiencia de formación de 42 profesores y profesoras egresadas, además de 7 académicos formadores. Los resultados muestran que mientras para los docentes universitarios la formación inicial debe privilegiar una mirada afectiva-actitudinal de la diversidad escolar, para el profesorado egresado

3 Consejo de Rectores de las Universidades Chilenas que reúne a las veinticinco principales universidades estatales y privadas. 
el hincapié debe estar puesto en una perspectiva cognitiva-pedagógica para atender adecuadamente la diversidad.

Ambas investigaciones en conjunto muestran que existen no solo dificultades desde el punto de vista de la formación inicial en torno a la diversidad, sino que además existen discontinuidades y tensiones en la manera de pensar y diseñar el espacio formativo entre los profesores formadores y los profesores egresados. En la literatura se han descrito estas tensiones señalando que el concepto de diversidad o multiculturalidad se usa para proponer proyectos educativos que difieren en aspectos tales como las metas, la población objetivo, las implicaciones curriculares, entre otros (Montecinos, 2011).

\section{MODELOS DE GESTIÓN DE LA DIVERSIDAD}

Aun cuando hasta el momento hemos hecho referencia a la diversidad en la escuela de manera genérica e incluso monolítica, existen diferentes concepciones y disposiciones hacia la diversidad, las que dan lugar a diferentes modelos de gestión. De esta manera, la forma en el profesorado gestiona la diversidad varía en función de las bases filosóficas, de las funciones educativas así como de los principios ético-políticos que estén a la base de sus procesos educativos (Essomba, 2006).

Tomando como evidencia la literatura internacional es posible identificar la existencia de diferentes modelos y enfoques utilizados tanto por las escuelas como por el profesorado para gestionar la diversidad (Dietz,2012). Autores pertenecientes a diversos contextos académicos y geográficos han confeccionado tipologías que sistematizan dichos enfoques y modelos (Aguado Odina, 2004; Banks, 1986; Besalú, 2002; Hannoun, 1992; Muñoz Sedano, 1998; Sleeter y Grant, 2009). Esta investigación ha utilizado los desarrollos de Sleeter y Grant (2009), ya que es fruto de un trabajo de sistematización de enfoques de educación multicultural por parte de los autores, constituyéndose en sí misma en una taxonomía representativa de dichos enfoques existentes.

Sleeter y Grant (2009) desarrollan una taxonomía que presenta cinco modelos de gestión de la diversidad en el ámbito educativo. Cada uno de ellos sintetiza la combinación de diferentes bases filosóficas, funciones educativas y principios ético-políticos asociados a los procesos educativos.

\section{Enseñar al alumnado excepcional y culturalmente diferente}

Corresponde al primer modelo de gestión y plantea que de cara a abordar exitosamente la diversidad en las aulas, el profesorado debe realizar ajustes pedagógicos que permitan la implementación de medidas compensatorias. Estas medidas persiguen en primer lugar nivelar los déficits - de conocimientos, de habilidades, de valores y/o procedimientos - de aquellos estudiantes diferentes, y en segundo lugar, utilizar dichas medidas compensatorias como una base para acortar las brechas entre el alumnado y el currículum de la escuela.

En la medida que los dispositivos compensatorios no solo tienen un sentido aditivo sino que además operan sustrayendo componentes que distorsionan o entorpecen el funcionamiento del alumnado (Jiménez, Lalueza y Fardella, 2017), 
resulta evidente el sentido asimilacionista y conservador de este enfoque de gestión de la diversidad (Jenks, Lee y Kanpol, 2001). Desde este enfoque, el éxito del proceso de adaptación-asimilación permite nivelar y habilitar al alumnado diferente para apropiarse e internalizar, con similares oportunidades y posibilidades de éxito, la propuesta educativa hegemónica de la escuela (Gorski, 2006, 2009).

\section{Promover relaciones humanas armónicas entre el alumnado}

Desde este segundo modelo la diversidad en la escuela es una dimensión que debe ser abordada por el profesorado con especial énfasis en el mejoramiento de la convivencia y los climas de aula, de modo de privilegiar el desarrollo de relaciones interpersonales armónicas entre los actores educativos. Se visualiza la diversidad como una oportunidad para el desarrollo de habilidades individuales (autoconcepto, confianza personal) así como competencias en el plano social (eliminación de prejuicios, modificación de estereotipos) que en conjunto, tributan al logro de una mayor simetría y equilibrio al interior de las aulas (Montecinos, 2011).

Este enfoque supone un progreso parcial de la ecuación déficit-compensación del modelo anterior y presenta una mayor sintonía por tanto con una perspectiva liberal de conceptualizar la ecuación entre diversidad y educación (Jenks, Lee y Kanpol, 2001). No obstante, el enfoque de relaciones humanas continúa evidenciando de manera subyacente la supremacía de la cultura hegemónica con relación al alumnado diferente, en la medida que bajo este modelo las prácticas educativas en la escuela permanecen mayoritariamente inalterables (Jiménez, 2012, 2013).

\section{Trabajo de grupo único}

Focalizar el trabajo de la escuela en un colectivo minoritario en particular es la finalidad de este tercer modelo de gestión de la diversidad. ${ }^{4}$ Este trabajo permite visualizar y comprender a dicho grupo, en su relación con la cultura hegemónica dentro del contexto educativo en particular. En la medida que el foco de atención de la relación entre estos grupos minoritarios y los grupos pertenecientes a la cultura dominante es sobre la base de la existencia de experiencias de discriminación, opresión y subordinación, los estudios de grupo único inauguran una primera modalidad de educación multicultural crítica (Jenks, Lee y Kanpol, 2001), que al ir más allá los enfoques asimilacionistas y de convivencia armónica, comienzan a conectar el rol de la escuela con principios de justicia social (Dover, 2009; Sleeter, 2013).

En tanto los estudios de grupo único buscan promover la comprensión del alumnado tanto desde un punto de vista histórico como contemporáneo de las condiciones de opresión, corresponde a un enfoque que define la escuela como un espacio desde el cual luchar contra las desigualdades sociales y las formas en que éstas se reproducen y actualizan al interior de los contextos educativos.

4 Alumnado con necesidades educativas especiales, alumnado perteneciente a minorías étnicas y/o alumnado inmigrante son algunas de las elecciones posibles de grupo único. 


\section{Educación multicultural}

Constituye un segundo modelo crítico de gestión de la diversidad, que va un paso más allá del enfoque de grupo-único. Este avance obedece a que el trabajo del profesorado apela a una reformulación global de los procesos educativos de la escuela. En el horizonte de combatir la discriminación hacia determinados estudiantes, provee oportunidades educativas de manera equitativa - no necesariamente igualitarias - y lucha por la justicia social de todos los grupos/colectivos presentes en la escuela (Montecinos y Ríos, 1999).

Este enfoque busca que el profesorado reconozca como parte de su ejercicio profesional el trabajo en torno a temas de poder y privilegio al interior del aula, alterando una mirada hegemónica de los contenidos, actividades y evaluaciones tradicionales utilizadas; es un llamado a enseñar sobre y en contra de la injusticia, garantizando en consecuencia la equidad educativa para todo el alumnado (Grant y Sleeter, 2006).

\section{Educación multicultural-reconstruccionista social}

Corresponde al último enfoque y plantea la necesidad que el profesorado vincule su rol con el desarrollo de procesos educativos que trasciendan el perímetro escolar, planteando la educación como un espacio de formación de estudiantes comprometidos activamente con el cambio social y con el fortalecimiento de una ciudadanía activa y participativa.

Una modelo de gestión multicultural-reconstruccionista ubica el trabajo del profesorado como una práctica para cuestionar el orden existente y para estimular entre sus participantes la reflexión en torno a formas posibles de cambiar las estructuras sociales que promueven ciertas lógicas, ciertas relaciones y determinados principios éticos y axiológicos basados en la desigualdad, la discriminación y la opresión sociocultural (Sleeter, Montecinos y Jiménez, 2016).

\section{PREGUNTAS DE INVESTIGACIÓN}

Los diversos planteamientos revisados fundamentan la necesidad de avanzar en el desarrollo de competencias profesionales para una exitosa gestión de la diversidad. Una gestión de la diversidad que en sintonía con las actuales demandas y desafíos del sistema educativo por resolver las desigualdades de aprendizaje y participación de ciertos estudiantes, contribuya a hacer de nuestras escuelas espacios educativamente más justos y pluralistas.

Una manera de avanzar en materia de formación inicial en torno al desarrollo de competencias para atender a la diversidad pasa por desarrollar investigaciones que puedan profundizar, por una parte, en cuáles son las demandas para gestionar la diversidad que le hace el sistema escolar a los docentes y por otra, en qué medida los programas de formación inicial acogen estas demandas. A través de la exploración de estos dos aspectos, se pueden levantar recomendaciones que sean de utilidad para la renovación curricular y para la identificación de nuevas competencias necesarias para el ejercicio profesional. 
De manera más específica la investigación busca responder las siguientes preguntas las siguientes preguntas:

- ¿Cuáles son las demandas que el sistema escolar está generando en materia de diversidad hacia los profesores?

- ¿Cómo gestionan la diversidad los profesores novatos? y ¿cómo se relaciona esa manera de gestionar la diversidad con la formación recibida en pedagogía?

- ¿Cuáles son las principales dificultades y oportunidades que los profesores visualizan en materia de diversidad en la escuela y cómo las relacionan con su formación recibida?

- ¿Cuáles son las competencias profesionales que la formación inicial de profesores desarrolla en materia de atención a la diversidad?

\section{METODOLOGÍA}

\section{PRODUCCIÓN DE DATOS}

Para dar respuesta a las preguntas de investigación se realizó un estudio de casos múltiple que contempló la realización de doce entrevistas en profundidad y veinte acompañamientos de aula a ocho profesores novatos de lenguaje y comunicación, vale decir, cuya experiencia profesional no fuese superior a cinco años. La muestra estuvo compuesta por siete mujeres y un hombre cuyas edades fluctúan entre los 23 y los 32 años. Para la realización de entrevistas y acompañamientos se utilizó un consentimiento informado visado por el comité de bioética de la universidad patrocinadora de esta investigación, donde se explicitó el carácter voluntario de participación, así como la confidencialidad y anonimato en el manejo de los datos. Todos los participantes son egresados de una universidad privada de la Región de Valparaíso y han sido formados con los mismos planes de estudio. Con relación al tipo de escuela, dos de ellos trabajaban en escuelas públicas, tres en escuelas privadas $\mathrm{y}$ tres en escuelas con financiamiento mixto.

\section{ANÁLISIS DE DATOS}

Tomando como referentes los planteamientos de la Grounded Theory (Glaser y Strauss, 1967) y mediante la técnica de análisis del contenido (Denzin y Lincoln, 1994; Krippendorff, 1990), se procedió a la codificación abierta del material textual de las transcripciones de entrevistas y observaciones de aula, con la ayuda del software Atlas.ti. Este procedimiento de primer nivel permitió la creación de cinco categorías analíticas de segundo orden $(\mathrm{C} 1, \mathrm{C} 2, \mathrm{C} 3, \mathrm{C} 4$, $\mathrm{C} 5$ ), que son las que han permitido organizar los resultados que se presentan a continuación.

\section{RESULTADOS}

Los resultados evidencian la predominancia de dos enfoques claves en la gestión de la diversidad por parte del profesorado. Cada uno de estos enfoques 
tiene a la base una conceptualización (C1) de la naturaleza de la diversidad, y al mismo tiempo formas de gestión (C2) coherentes con dichas conceptualizaciones. A su vez, las conceptualizaciones que hacen los profesores acerca de la diversidad establecen determinadas demandas (C3) hacia las prácticas educativas que estos desarrollan, mientras que las formas de gestión implementadas por el profesorado se encuentran con determinados factores constrictores $(\mathrm{C} 4)$ y factores promotores (C5) que tensionan o favorecen la entrega de una adecuada atención a la diversidad.

Tanto las conceptualizaciones predominantes como sus consecuentes formas de gestión presentan desafíos formativos (C6) - percibidos o no - para una gestión holística y no reduccionista de la diversidad, en línea con los planteamientos de la justicia social en el ámbito educativo. Finalmente, en algunos casos se entregan además recomendaciones (C7) para el mejoramiento de la formación inicial docente en la gestión de la diversidad en el ámbito educativo. El modelo explicativo planteado se resume en la siguiente Figura 1.

\section{ENFOQUE DE EDUCAR A LOS DIFERENTES}

El primer enfoque que predomina discursivamente entre el profesorado y que se expresa al mismo tiempo en sus prácticas de aula, corresponde a aquel donde la atención a la diversidad en la escuela es asumida como la educación del alumnado excepcional y culturalmente diferente (Grant y Sleeter, 2006), donde la diferencia refiere principalmente a la existencia de algún tipo de déficit con relación a la norma y/o estándares vinculados al desarrollo de aprendizajes. Al interior de este primer enfoque se evidencian dos modalidades claramente diferenciadas: una asociada a

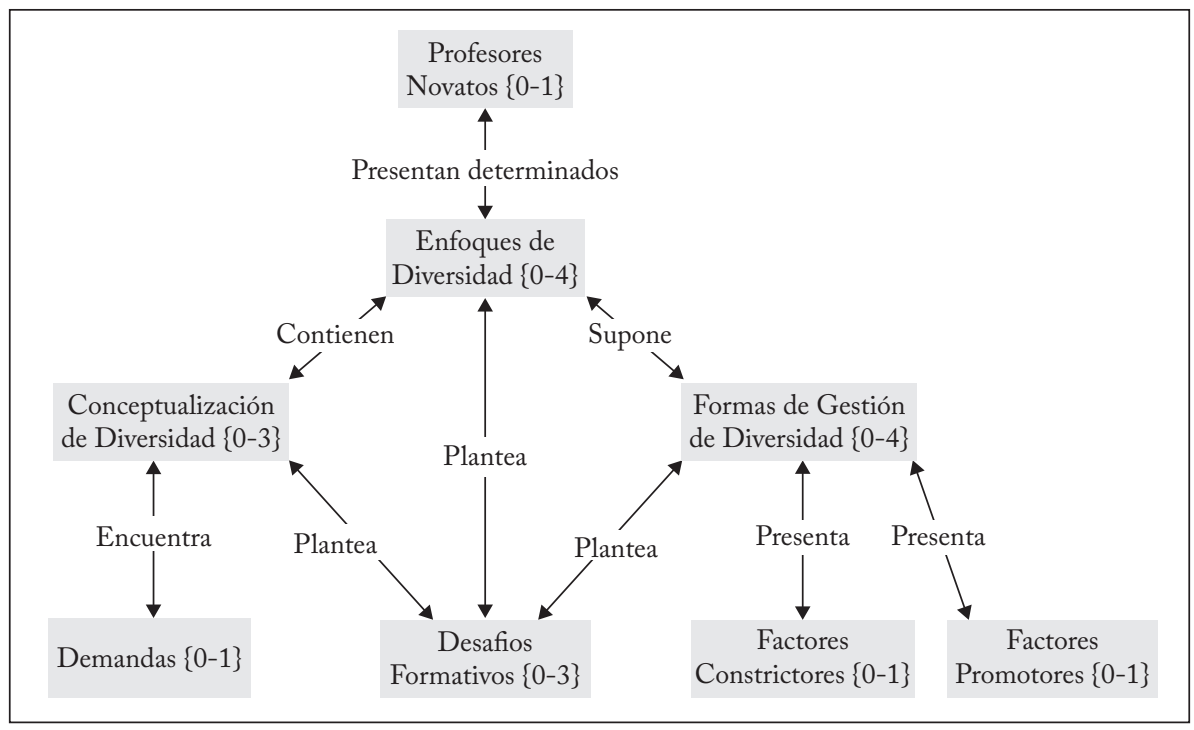

Figura 1 - Relaciones entre categorías emergentes

Fonte: Banco de dados de la encuesta.

Elaboración propia. 
las necesidades especiales del alumnado y otra asociada a la condición de vulnerabilidad de éste. ${ }^{5}$

\section{Necesidades educativas especiales como déficit}

Cuando la diversidad es asociada a diferencias académicas de los estudiantes (C1), predomina una mirada problemática ya que supone la implementación de ajustes curriculares y metodológicos para diversificar los procesos de enseñanza y aprendizaje. Esto además de suponer un trabajo adicional y un desgaste profesional, no siempre tiene los resultados que los profesores esperan. Como consecuencia de ello existe la creencia profesional que trabajar en contextos homogéneos es más simple y más "rentable" que en contextos con mayor diversidad entre los estudiantes.

[...] hay que bajar sí mucho el contenido, muchísimo, la forma de tratar a los niños también no es la misma que la que uno se pueda imaginar, aunque sean de los cursos iguales. $\mathrm{O}$ sea un quinto de este tipo de realidad es quizás una mezcla de bastantes niveles entonces en la sala hay mucha heterogeneidad de edad, de vivencias y eso dificulta mucho lo que uno hace como profesor porque los intereses varían demasiado, o sea desde el que quiere estar pololeando en quinto básico hasta el que de verdad es un niño todavía en quinto básico. Entonces hay que lidiar con todas esas realidades y más, hay que ser más asistente social, hay que tener otros roles aparte de docente. Y yo no sentía que tenía esas capacidades para estar ahí. O sea, ni siquiera, claro, no tenía ni las capacidades ni me sentía con las ganas de hacerlo, sentía que no había estudiado para eso. (Entrevista profesor 7, escuela privada)

Desde la literatura (Sleeter y Grant, 2009) y tal como ha sido documentado en este trabajo a partir de los discursos del profesorado, este enfoque sobre la diversidad supone la necesidad de realizar ajustes a los procesos educativos ordinarios desde una lógica compensatoria (C2) (Cochran-Smith, 2010), forma de gestión que es vista como una oportunidad de hacer frente a los hándicaps y las deficiencias que estos estudiantes presentan con relación a la mayoría.

De esta manera, las principales demandas en torno a diversidad se concentran en la dimensión didáctica de la labor educativa (C3), más específicamente en torno a las diferencias de rendimiento y a las dificultades de aprendizaje que presentan determinados estudiantes. Los casos más frecuentes y problemáticos corresponden a estudiantes que son percibidos con algún tipo de necesidad educativa especial y que presentan generalmente alguna modalidad de trastorno ligado al aprendizaje. En tales casos los profesores señalan que los estudiantes no son capaces de seguir con normalidad el ritmo y nivel de la clase, situación que los va retrasando y que les impide rendir con éxito en las pruebas estandarizadas.

5 Para los efectos de este trabajo y por razones de espacio se presentarán únicamente los resultados de la primera modalidad relativa a las necesidades educativas especiales, de manera de incluir también resultados del segundo enfoque predominante (relaciones humanas), complejizando los análisis y las discusiones finales. 
[...] otros muchos tienen problemas de aprendizaje, muchos problemas de aprendizaje, yo tengo muchos niños, tengo en mi curso por lo menos 5 niños de integración. Que tienen problemas de aprendizaje, que les cuesta... o tienen déficit atencional o tienen otros modos de aprendizaje que no se asemejan al grupo curso y yo no puedo estar haciendo malabares porque yo tengo tantos niños, tengo un caso de un niño limítrofe también. La regla es de 5 niños PIE por curso, hay un curso que tiene como 10. Por ley el máximo son 5 niños PIE por curso y hay un curso que se escapa de eso, que no hemos podido regularizar la situación y bueno eso po. (Entrevista profesor 1, escuela pública)

Estas demandas percibidas son gestionadas de manera problemática por el profesorado ya que como evidencia el trabajo etnográfico de acompañamiento de aula, se manifiesta un claro predominio de procesos de enseñanza de estilo homogéneo (C4), donde las actividades, metodologías, formas de participación, niveles de exigencia así como formas de evaluación se realizan bajo una modalidad única para todo los estudiantes.

Nos vamos al aula y el profesor me presenta, saluda y les pide a todos que se pongan la capa. Un alumno aprovecha mi presentación como psicólogo y pregunta si aprovecharé de "revisar" a otro alumno, lo que provoca risas en el resto (menos en el aludido). Es una sala muy pequeña y hay solo once estudiantes que se ubican en dos filas hacia el pizarrón. Luego de ello el profesor entrega una ficha de actividad en la que los estudiantes deben leer un artículo denominado "la alimentación" y responder tres preguntas que son de comprensión lectora. La actividad está planteada para que sea realizada individualmente, es la misma para todos y no presenta ni instrucciones ni ayudas diversificadas. Mientras los estudiantes van terminando entregan su trabajo al profesor.Éste corrige los trabajos con el mismo criterio para todos y luego lee en voz alta las notas y explica las respuestas correctas. Frente a una pregunta que presentó más errores recuerda que fue un tema que habían trabajado anteriormente. Luego de ello y sin más revisión de lo hecho pide a los estudiantes que saquen sus cuadernos para pasar a la siguiente actividad. (Acompañamiento de aula profesor 8 , escuela particular-subvencionada)

Al mismo tiempo se observa que los procesos de enseñanza-aprendizaje son, salvo contadas excepciones, de carácter individual (C4) donde cada estudiante trabaja de manera independiente. Cuando por el contrario las actividades se realizan de manera grupal, la tendencia es a que existan agrupamientos espontáneos entre los estudiantes $(\mathrm{C} 4)$, dando origen a grupos fuertemente marcados por el género y donde además se observan algunos estudiantes que quedan excluidos de la formación de grupos. Generalmente ambas situaciones (polarización y exclusión) no son temas que generen demasiada preocupación a los profesores.

La profesora me recibe en el hall de entrada de la escuela y luego nos vamos a la sala de profesores, para finalmente dirigirnos al aula del $7^{\circ} \mathrm{C}$. Ingresamos y ella pide que todos se paren y se produce el "ritual" de saludar a la profesora. 
Como primera instrucción les pide que separen los bancos por lo que se produce un cambio en la organización de la sala, quedando seis filas de asientos. Mientras los estudiantes ordenan la nueva disposición del aula y como estoy a su lado, me comenta que estos alumnos son "frescos" porque saben que tienen que separar los asientos pero no lo hacen. No me explica por qué. Una vez ubicado en mi lugar al fondo del aula y aprovechando un momento de distensión de la clase, le pregunto a una alumna que está al lado mío acerca de por qué los separan y me dice "porque hablamos mucho" [...] En el recreo aprovecho de preguntarle por el tema de la separación de sillas y ella un poco sorprendida por mi pregunta me dice "porque tienen que estar separados" como si eso fuese lo normal, y luego agrega que son muy chicos y que si los junta, se ponen a conversar y no trabajan. (Acompañamiento de aula profesor 3, escuela privada)

Independiente del tipo de escuela (pública, privada o subvencionada) estas diferencias del alumnado en cuanto al rendimiento y dificultades de aprendizajes, generan presiones al profesorado para el cumplimiento de estándares de logro y para el cumplimiento de objetivos y contenidos curriculares revisados. Esto la mayoría de las veces es percibido por el profesorado como un elemento de constricción de la diversidad que tensiona su quehacer docente, ya que por un lado supone un trabajo más personalizado y diversificado hacia ciertos estudiantes que requieren un trato diferente (para el cual no se tienen las herramientas), pero al mismo tiempo se señala la necesidad de desarrollar habilidades para rendir en pruebas estandarizadas (SIMCE y Prueba de Selección Universitaria - PSU) que no toman en cuenta estas diferencias.

Sí, lo hago porque quiero seguir en este colegio [usar pruebas estandarizadas], lo hago porque necesito que me vaya bien en la prueba SIMCE, pero en mis clases, no es muy coherente, pero si bien en la prueba SIMCE y en la prueba global, esa es la forma de evaluar poh, porque quieren que a los cabros les vaya bien en esas cosas, ¿cachai? [...] todos tenemos capacidades distintas y siento que con eso se pierde la capacidad diferente que puedes tener. Tú y yo o mis talentos los voy a perder en esa prueba. Tengo alumnos que les encanta dibujar y eso nunca se va a ver en la PSU o en el SIMCE y que son fantásticos dibujando. O no sé poh, tengo un chiquillo que le cuesta harto, pero el otro día, no sé poh, pasó un acontecimiento. Pasó que murió una colega de nosotros y este chiquillo estaba súper afectado y me dijo “isabe qué tía?, le voy a hacer una canción"y en cinco minutos este cabro que me cuesta un año enseñarle gramática me hizo una canción súper linda, entonces esas cosas jamás las vamos a ver poh. (Entrevista profesor 3, escuela privada)

Los resultados evidencian que este enfoque basado en la idea de déficit (Chubbuck,2010), supone por parte del profesorado la implementación de medidas adaptativas provenientes preferentemente del propio alumnado. Esto ya que, como se ha observado anteriormente, el profesorado reconoce la falta de herramientas teórico-prácticas para impulsar cambios pedagógicos y didácticos en sus propias prácticas docentes, vale decir, hacer frente a las barreras que el propio ejercicio profesional establece en los procesos de enseñanza y aprendizaje (Ainscow, 2004; Booth y Ainscow, 2002). 
Sin embargo, los profesores también reconocen ayudas institucionales que actúan como factores promotores de la diversidad y que permiten sortear la falta de herramientas profesionales antes descritas. La existencia de ayudas de atención a la diversidad de carácter institucional (C5) es percibida en consecuencia como un recurso necesario, el cual sin embargo no siempre está disponible en las escuelas o en los niveles en que los profesores se desempeñan, o bien existen procedimientos excesivamente burocratizados que ralentizan una oportuna atención a la diversidad de necesidades de los estudiantes.

Los colegios deberían también tener a los profesionales adecuados para todos los niveles donde vayan a haber alumnos con evaluación diferencial o algún tipo de diversidad. Tendrían que haber psicopedagogos, psicólogos que te ayudaran po. Entonces como te digo con mi pololo siempre tenemos esta discusión porque él está encargado en su colegio de hacer talleres para los profes, de ayudarlos, de ayudar en cierto material, no sé. Y nosotros no tenemos nada de eso [...] y yo creo que yo lo expreso, sobre todo esto, pero yo creo que no lo dices muy abiertamente para no quedar mal, que no sabes hacerlo porque aquí se da mucho eso entonces eso me gustaría, que si se va a tomar como medida que se haga bien y que se implementen las medidas necesarias para ayudar a las personas que lo necesitan. (Entrevista profesor 3, escuela privada)

Finalmente, la valoración que los profesores hacen de su formación profesional cambia sustancialmente en virtud de las dimensiones evaluadas. Desde el punto de vista disciplinar existe consenso en considerar la formación como adecuada y sólida, al punto de contar con competencias de la especialidad que están por sobre las necesidades y requerimientos de los contextos escolares. Si bien esto genera seguridad y confianza profesional, al considerar la dimensión pedagógica de su profesión docente, la valoración es de una formación deficiente e incompleta (C6) que no logra entregar las herramientas necesarias para abordar la complejidad de situaciones que ocurren durante el ejercicio profesional. Los profesores cuestionan principalmente una formación centrada en lo teórico y en una enseñanza modélica educativamente inexistente (C6).

La formación inicial, yo estoy súper agradecida de mi formación y de la universidad, de todas las puertas que me ha abierto y que me sigue abriendo hasta el día de hoy, pero siento que sí hay un vacío en ese tipo de cosas que estamos un poco solos los profesores en este aspecto, me gustaría que cambiara. Yo creo que por eso mismo accedí a esta entrevista como con esa misma motivación también de que esta información llegue y que pudieran cambiar de alguna forma las cosas para que los demás profesores sí tengan esa preparación que nosotros no tenemos porque al final yo como profesora tengo ahora, no me siento preparada para esto, para todas estas dificultades porque la universidad muchas veces te propone y te hace hacer clases para cursos ideales donde tú no tienes problemas, donde tú no tienes alumnos con dificultades, donde tú no tienes alumnos distintos, entonces esa es mi metodología, la que me dio la universi- 
dad: hacer clases perfectas para alumnos perfectos. Y no es así poh. (Entrevista profesor 3, escuela privada)

Sobre la base de estas dificultades y de las lagunas formativas, los propios profesores entregan recomendaciones (C7) de cómo se podría fortalecer la formación inicial de manera de formar profesionales con mayores herramientas para una adecuada gestión de la diversidad en la escuela.

Más ramos de pedagogía, no sacaría los ramos de literatura y lingüística porque eso es un sello de nuestra carrera que nosotros salimos con harto conocimiento y de buena calidad. No los sacaría o quizás sacaría como uno que era muy mula, pero más pedagogía, quizás más prácticas también, quizás más prácticas profesionales o más ramos que intervengan en las escuelas, más intervención. Sí. Porque uno llega en pañales. (Entrevista profesor 1, escuela pública)

\section{ENFOQUE DE RELACIONES HUMANAS}

Cuando la diversidad es vista por los profesores más allá de la idea de déficit y su impacto en la dimensión didáctica, emerge un segundo enfoque de atención a la diversidad, un enfoque centrado en las relaciones humanas (Grant y Sleeter, 2006). Ver la diversidad desde las relaciones humanas supone para los profesores que uno de los funciones básicas de la escuela es el de enseñar a convivir con la diferencia, siendo los profesores en consecuencia los responsables de promover habilidades de tolerancia, respeto y no discriminación hacia la diversidad.

Cuando los profesores perciben la diversidad desde la óptica de las relaciones humanas, predomina una conceptualización de la diversidad a partir de las diferencias culturales ${ }^{6}(\mathrm{C} 1)$, que a diferencia del enfoque anterior centrado en el déficit, es valorado en términos de mayor neutralidad; estas diferencias culturales no son percibidas per se como algo negativo o deficitario, sino como algo natural y propio de las relaciones humanas actuales.

Tiene que ver con las nacionalidades [la diversidad en el aula], con los alumnos extranjeros que tenemos, tenemos varios, pero para ellos no es tema, para nada [...] sí, no es tema, puede ser porque la mayor parte... Tengo un alumno que se va a Costa Rica entonces levanté la mano y pregunté "quién ha ido a otro país" y el 98\% del curso, y no hablamos de Argentina o Brasil, hablamos de París. Entonces para ellos no es tema, la diversidad sexual tampoco es tema porque yo he visto alumnos, incluso profesores que tienen una marcada tendencia homosexual, para ellos no es tema. Creo que podría ser más tema lo de las religiones [...]. (Entrevista profesor 6, escuela privada)

6 Para los efectos de este trabajo y por razones de espacio se presentarán únicamente los resultados de la primera modalidad relativa a las necesidades educativas especiales, de manera de incluir también resultados del segundo enfoque predominante (relaciones humanas), complejizando los análisis y las discusiones finales. 
Esta viñeta refleja que entre los profesores prevalece la creencia que una adecuada atención a la diversidad es aquella que privilegia un trato igualitario $(\mathrm{C} 2)$ que se expresa en no mostrar preferencias hacia determinados estudiantes y en no "visibilizar" con demasiada notoriedad dichas diferencias. Esto deja en evidencia a su vez que para los profesores solo son relevantes aquellas diferencias que tienen un impacto negativo en el ámbito del aprendizaje; las que no, son pasadas por alto y se reconocen más bien como parte del ámbito privado de las personas y como tal, como dimensiones que no necesariamente correspondería trabajar en el espacio público escolar.

En general yo no distingo oportunidades porque yo le digo a mis alumnos que "si le doy una oportunidad a usted tengo que dársela a todos" y no puedo hacerlo entonces ahí los niños entienden igual, ellos entienden [...] a veces al niño también tú le tienes un trato especial distinto, en general, uno trata de no hacerlo tan notorio porque todos te reclaman afecto, todos te reclaman atención y se dan cuenta también si uno tiene favoritismos. Yo, este trato que yo te digo no lo traduzco en favoritismos, en las clases ni nada. (Entrevista profesor 1, escuela pública)

A diferencia del enfoque anterior de educar a los diferentes donde existe una forma de gestión centrada en la incorporación de la diversidad, un enfoque de relaciones humanas privilegia un estilo de gestión basado en la problematización de la diversidad (C2), Esto ya que más que prevenir que determinadas diferencias se constituyan en barreras - algo propio de un modelo de incorporación de la diversidad -, la problematización de la diversidad lo que busca es intencionar que determinados procesos/reflexiones/discusiones ocurran al interior del aula, por lo que tiene un sentido más promocional de relaciones armónicas y respetuosas entre todos los miembros de la comunidad educativa.

Yo lo hago [trabajar la diversidad] desde la literatura o desde la narración de algunos cuentos. Nosotros como departamento de lenguaje tratamos de tener un plan lector que aborde temáticas que ellos no estén acostumbrados a ver, por ejemplo, nos hicieron sacar un libro "Violeta se va a los cielos" que era de Violeta Parra, y mostraba una realidad que quizás no era a la que ellos estaban acostumbrados, pero sí por ejemplo, como vemos el contenido de otras tipologías textuales, por ejemplo, la noticia o la poesía uno va mostrando otras realidades [...] Les dije "oye la realidad es mucha más amplia que esto, date cuenta" y yo no sé incluso, cuando pasó lo de Valparaíso nosotros somos de Valparaíso con otra colega y nos preguntaban "se le quemó la casa o algo así... no, no se me quemó a mí, pero hay muchas más otras personas que sí se les quemó". Entonces, y se habla de ese tema y se trata de abrir un poco el ojo que tienen, pese a lo mal visto que puede ser por los apoderados o los directivos. (Entrevista profesor 7, escuela privada) 
En la medida que el trabajo de promover el respeto a las diferencias y la igualdad de oportunidades a través del trabajo valórico no supone una dimensión problemática por parte de los profesores noveles, no existen factores que constriñan de manera clara el trabajo en torno a estas temáticas (C4) ni demandas explícitas para el mejoramiento del trabajo en torno a este ámbito (C3). Esta misma explicación corre para el hecho que no exista con la misma contundencia que en el enfoque anterior propuestas para el mejoramiento de la formación docente (C7).

\section{DISCUSIONES Y RECOMENDACIONES}

\section{PARA LA FORMACIÓN INICIAL DEL PROFESORADO}

En virtud de los resultados presentados que evidencian una predominancia de enfoques basados en el déficit y el fortalecimiento de las relaciones humanas, y a la luz de los planteamientos de una educación para la justicia social (Dover, 2009; Gorski, 2006, 2009; Sleeter y Grant, 2009), los desafíos formativos del profesorado se ubican en dos líneas complementarias.

Por un lado se hace necesario fortalecer las competencias pedagógicas en aquellos ámbitos reconocidos como demandas directas, entre los que se encuentra el desarrollo de estrategias diversificadas de enseñanza y sus correspondientes instancias de evaluación. Sin embargo y en la medida que las dificultades académicas son atribuidas por el profesorado a déficit internos de los estudiantes, resulta conveniente promover una perspectiva inclusiva de atención a la diversidad que reconozca el origen sistémico de las dificultades, así como la existencia de barreras institucionales hacia el aprendizaje y la participación del alumnado (Ainscow,2004; Booth y Ainscow, 2002).

Mejorar las competencias para resolver las dificultades académicas de estudiantes diversos no solo requiere de la dotación de una mayor formación pedagógica y didáctica, sino que requiere de manera complementaria la implementación de espacios reflexivos para la revisión y el cuestionamiento de creencias estereotipadas en torno a las formas en que se producen los aprendizajes. Estas creencias, la mayoría de las veces fuertemente arraigadas pueden dificultar la adquisición de una perspectiva inclusiva y de justicia social en torno a la diversidad (Sleeter, 2013). Superar un enfoque de déficit por ejemplo requiere no solo que el profesorado cuenta con mayores herramientas metodológicas para trabajar determinados contenidos, sino además que cuente con instancias formativas que le permita cuestionar la las explicaciones que naturalizan e internalizan las dificultades de aprendizaje de los estudiantes.

En segundo lugar se evidencia la necesidad de incluir espacios formativos donde puedan ser trabajadas temáticas que si bien no constituyen demandas directas del profesorado, corresponden a desafíos detectados que permitan el desarrollo de un enfoque global de atención a la diversidad. Avanzar desde una atención a la diversidad restringida al ámbito pedagógico hacia una perspectiva de justicia social de la diversidad, supone considerar espacios formativos que incluya un trabajo en torno a:

- Problematizar la forma en que son conceptualizadas y valoradas las diferencias individuales así como los entornos socioculturales y familiares de 
pertenencia del alumnado, ampliando y complejizando las perspectivas predominantes de déficit (Cochran-Smith, 2004).

- Realizar la naturaleza situada de los aprendizajes (Cole, 1996; Vygotsky, 1978), estableciendo la importancia que las experiencias y los conocimientos - culturales-previos juegan en el desarrollo de éstos - . Al mismo tiempo, se hace necesario ampliar el rol que dichos conocimientos y experiencias desempeñan, ampliando su utilidad más allá de su uso en el desarrollo de nuevos aprendizajes, reconociendo su importancia tanto en la socialización del alumnado como en el reconocimiento, la expresión y el mantenimiento de su babitus sociocultural (Esteban-Guitart, 2011).

- Promover un sentido formativo de la evaluación (Agut, 2010) que no solo permita "calibrar aprendizajes", sino que a su vez entregue información útil para la identificación y el establecimiento de ayudas ajustadas al alumnado e información relevante para la evaluación del propio desempeño docente. Se hace necesario además, reconocer el impacto negativo que puede tener el uso - y abuso - de evaluaciones estandarizadas, convirtiéndose en instrumentos que pueden promover más que evitar las desigualdades entre el alumnado (Stobart, 2010).

- Desarrollar estrategias curriculares de gestión y atención a la diversidad que vayan más allá de prácticas de consideración y reconocimiento de las diferencias socioculturales (estilos y ritmos de aprendizaje, género, pertenencia cultural, entre otras), incluyendo además estrategias que apunten a la problematización crítica de la diversidad. Este trabajo de problematización permitiría a través de una mayor programación docente, transformar las desigualdades que se legitiman y reproducen en el espacio educativo (Francis y Le Roux, 2011; North, 2008). Avanzar en la problematización de la diversidad escolar permitiría por tanto formar profesores desde un modelo multiculturalista de gestión de la diversidad, que sean capaces de cuestionar las diferencias y develar los mecanismos educativos que contribuyen a su reproducción (Grant y Sleeter, 2006).

- Finalmente, promover una mirada de la escuela como un lugar desde el cual luchar contra las desigualdades, promoviendo diversos niveles de compromiso político en el ejercicio docente, evitando de esta manera una labor docente neutral y aséptica que centralice su función en el desarrollo competencias de la disciplina en cuestión (Hickling-Hudson, 2004; Lazar, 2013).

Este conjunto de propuestas formativas que recogen tanto la voz de los propios profesores novatos así como las recomendaciones de la literatura especializada, se constituyen en insumos para la discusión actual en torno a los desafíos formativos del profesorado. Este trabajo investigativo ha evidenciado que uno de estos desafíos formativos pasa entonces por permitir que el profesorado visualice su rol docente como un agente-clave para la reconstrucción de nuestras sociedades, contribuyendo a resolver las inequidades de nuestro actual sistema educativo. 


\section{REFERENCIAS}

AGCE - Agencia de Calidad de la Educación. Informe nacional resultados Chile PISA 2012. Santiago: División de Estudios, Departamento de Estudios Internacionales, Agencia de Calidad de la Educación, 2014.

Aguado Odina, T.Investigación en educación intercultural. Educatio Siglo XXI, Murcia: Universidad de Murcia, n. 22, p. 39-58, 2004.

Agut, N. La evaluación en un modelo de escuela inclusiva.Aula de Innovación Educativa, Barcelona: Graó, n. 191, p. 42-44, 2010.

Ainscow, M. Desarrollo de escuelas inclusivas. Ideas, propuestas y experiencias para mejorar las instituciones escolares. Madrid: Narcea, 2004.

Avalos, B. Profesores para Chile, historia de un proyecto. Santiago: MINEDUC, 2002. . El nuevo profesionalismo: formación docente inicial y continua. In: Tenti, E. (Comp.). El oficio docente; vocación, trabajo y profesión en el siglo XXI. Buenos Aires: Siglo XXI Editores, 2006. p. 209-238.

. La inserción profesional de los docentes. Profesorado - Revista de Currículum y Formación del Profesorado, Granada: Universidad de Granada, p. 43-59, 2009.

BANKs, J. Multicultural education: development, paradigms and goals. In: BANKs, J. A.; Linch, J. (Eds.). Multicultural education in western societies. Londres: Holt, Rinehart and Winston, 1986. p. 12-28.

Besalú, X. Diversidad cultural y educación. Madrid: Síntesis, 2002.

Bоoтн, T.; Ainscow, M. Index for inclusion. Bristol. United Kingdom: Centre for Studies on Inclusive Education, 2002.

Сниввиск, S. Individual and structural orientations in socially just teaching: conceptualization, implementation, and collaborative effort. Journal of Teacher Education, Thousand Oaks, CA: Sage, v. 61, n. 3, p. 197-210, 2010.

Cochran-Smith, M. Walking the road: race, diversity and social justice in teacher education. New York: Teachers College Press, 2004.

. Toward a theory of teacher education for social justice. In: Hargreaves, A.; Fullan, M.; Hopkins, D.; Lieberman, A. (Eds.). Second International Handbook of Educational Change. New York: Springer, 2010. p. 445-467.

Cole, M. Cultural psychology: a once and future discipline. Cambridge, MA: Harvard University Press, 1996.

Denzin, N. K.; Lincoln, Y. S. Introduction: entering the field of qualitative research. In: . (Eds.). Handbook of qualitative research. Thousand Oaks, CA: Sage, 1994. p. 1-34.

Dietz, G. Multiculturalismo, interculturalismo y diversidad en educación. Una aproximación antropológica. México, DF: Fondo de Cultura Económica, 2012.

Dover, A. Teaching for social justice and K-12 student outcomes: a conceptual framework and reserch rewiev. Equity and Excellence in Education, United Kingdom: Taylor \& Francis, v. 42, n. 4, p. 506-524, 2009. 
Essombа, M. Liderar escuelas interculturales e inclusivas. Equipos directivos y profesorado ante la diversidad cultural y la inmigración. Barcelona: Graó, 2006.

Esteban-Guitart, M. Una interpretación de la psicología cultural: aplicaciones prácticas y principios teóricos. Suma Psicologica, Bogotá: Fundación Universitaria Konrad Lorenz, v. 18, n. 2, p. 65-88, 2011.

Francis, D.; Le Roux, A. Teaching for social justice education: the intersection between identity, critical agency, and social justice education. South African Journal of Education, Pretoria: Education Association of South Africa, n. 31, p. 299-311, 2011.

Glaser, B.; Strauss, A. Discovery of grounded theory. Chicago: Aldine, 1967.

Gorski, P. Complicity with conservatism: the do-politicizing of multicultural and intercultural education. Intercultural Education, United Kingdom: Taylor \& Francis, v. 17, n. 2, p. 163-177, 2006.

.What we're teaching teachers: an analysis of multicultural teacher education coursework syllabi. Teaching and Teacher Education, United Kingdom: Elsevier, n. 25, p. 309-318, 2009.

Grant, C.; Sleeter, C. Turning on learning: five approaches to multicultural teaching plans for race, class, gender, and disability. Upper Saddle River, NJ: Prentice-Hall, 2006. Hannoun, H. Els guettos de l'escola. Per una educació intercultural. Vic: EUMO Editorial, 1992.

Hickling-Hudson, A. Educating teachers for cultural diversity and social justice. In: Hernes, G.; Martin, M. (Eds.). Planning for diversity: education in multi-ethnic and multicultural societies. Paris: International Institute for Education Planning (UNESCO), 2004. p. 270-307.

IвÁ̃̃ez, N. El contexto interaccional y la diversidad en la escuela. Estudios Pedagógicos, Valdivia: Universidad Austral de Chile, v. 36, n. 1, p. 275-286, 2010.

Jenks, C.; Lee, J.; Kanpol, B. Approaches to multicultural education in preservice teacher education: philosophical frameworks and models for teaching. The Urban Review, New York: Springer, v. 33, n. 2, p. 87-105, 2001.

JimÉnEz, F.Violencia escolar en contextos educativos multiculturales: una aproximación desde los modelos de gestión de la diversidad cultural. Psicoperspectivas, Viña del Mar: Pontificia Universidad Católica de Valparaíso, v. 11, n. 2, p. 12-34, 2012.

. Discursos y prácticas educativas en la escuela multicultural. Una aproximación etnográfica a la escolarización de alumnado inmigrante y de culturas minoritarias. Tesis (Doctoral) - Universidad Autónoma de Barcelona. Barcelona, 2013.

Jiménez, F.; Fardella, C. Diversidad y rol de la escuela: discursos del profesorado en contextos educativos multiculturales en clave migratoria. Revista Mexicana de Investigación Educativa, México, DF: Consejo Mexicano de Investigación Educativa A.C., v. 20, n. 65, 2015.

Jiménez, F.; Lalueza, J.; Fardella, C. Aprendizajes, inclusión y justicia social en entornos educativos multiculturales. Revista Electrónica de Investigación Educativa, México: Universidad Autónoma de Baja California, v. 19, n. 3, p. 10-23, 2017. 
Krippendorff, K. Metodología de análisis de contenido: teoría y práctica. Barcelona: Editorial Paidós, 1990.

LAZAR, A. Degrees toward social justice teaching: examining the dispositions of three urban early-career teachers. The Urban Review, New York: Springer, v. 45, n. 5, p. 701-727, 2013. Miranda, C. Educación superior, mecanismos de aseguramiento de la calidad y formación docente: un debate pendiente en Chile. Estudios Pedagógicos, Valdivia: Universidad Austral de Chile, v. 33, n. 1, p. 95-108, 2007.

Montecinos, C. Desarrollo profesional docente y aprendizaje colectivo. Psicoperspectivas, Viña del Mar: Pontificia Universidad Católica de Valparaíso n. 2, p. 105-28, 2003.

. Propuestas para una educación que es multicultural. In: Williamson, G. C.; Montecinos, C. (Orgs.). Educación multicultural: práctica de la equidad y diversidad para un mundo que demanda esperanza. Temuco, Chile: Ediciones Universidad de la Frontera, 2011. p. 15-42.

Montecinos, C.; Ríos, F. Assesing preservice teachers'zones of concern and comfort with multicultural education. Teacher Education Quarterly, [S.1.: s.n.], n. 26, p. 7-24, 1999. Muñoz Sedano, A. Hacia una educación multicultural: enfoques y modelos. Revista Complutense de Educación, Madrid: Universidad Complutense de Madrid, v. 9, n. 2, p. 101-135, 1998.

North, C. What is all this talk about "Social Justice"? Mapping the terrain of education's latest catchphrase. Teachers College Record, New York: Columbia University, v. 110. n. 6, p. 1.182-1.206, 2008.

OECD - Organisation for Economic Co-Operation and Development. PISA 2012 results. Excellence through equity: giving every student the chance to succeed. [S.1.]: PISA, OECD Publishing, 2013. v. II.

Quilaqueo, D. Y.; Quintriqueo, S. Formación docente en educación intercultural para contexto Mapuche en Chile. Cuadernos Interculturales, Valparaíso: Universidad de Valparaíso, v. 6, n. 10, p. 91-110, 2008.

Sleeter, C. Teaching for social justice in multicultural classroom. Multicultural Education Review, United Kingdom: Taylor \& Francis, v. 5, n. 2, p. 1-18, 2013.

Sleeter, C.; Grant, C. Making choices for multicultural education: five approaches to race, class and gender. 6th ed. Hoboken, NJ: Wiley, 2009.

Sleeter, C.; Montecinos, C.; Jimenez, F. Preparing teachers for social justice in the context of education policies that deepen class segregation in schools: the case of chile. In: LAMPert, J.; Burnett, B. (Eds.). Teacher education for high poverty schools. New York: Springer Book Series, 2016. p. 171-191.

Stobart, G. Tiempos de pruebas: los usos y abusos de la evaluación. Madrid: Morata, 2010. Tenorio, S. Formación inicial docente y necesidades educativas especiales. Estudios Pedagógicos, Valdivia: Universidad Austral de Chile, v. 37, n. 2, p. 249-265, 2011.

Venegas, C. Hacia la innovación en la formación inicial docente para un desempeño exitoso en contextos alta vulnerabilidad social y educativa. Revista de Estudios y Experiencias en Educación, Concepción: Universidad Católica de la Santísima Concepción, v. 12, n. 23, p. 47-59, 2013. 
Vygotsкy, L. Mind in society. Cambridge, MA: Harvard University Press, 1978.

Zapata, R. Formación del futuro profesorado en diversidad cultural: Estudio de un caso en el contexto universitario chileno. Revista Digital Akadèmeia, [S.1.: s.n.], v. 2, n. 2, 2011.

\section{SOBRE LOS AUTORES}

Felipe Jiménez Vargas es doctor en psicología de la educación por la Universidad Autónoma de Barcelona (España). Profesor de la Pontificia Universidad Católica de Valparaíso (Chile).

E-mail: felipe.jimenez@pucv.cl

Carmen Montecinos Sanhueza es doctora en psicología educacional por la Southern llinois University Carbondale (EE.UU.). Profesora de la Pontificia Universidad Católica de Valparaíso (Chile).

E-mail: carmen.montecinos@pucv.cl

Recebido em 24 junho de 2015

Aprovado em 23 de maio de 2016 